\title{
The Efficacy and Safety of PD-1/PD-L1 Inhibitors in Combination with Conventional Therapies for Advanced Solid Tumors: A Meta-Analysis
}

\author{
Run-Cong Nie ${ }^{1},{ }^{1}$ Chong-Bang Zhao, ${ }^{2}$ Xiao-Wei Xia, ${ }^{2}$ Ying-Shan Luo, ${ }^{3}$ Ting Wu, ${ }^{1}$ \\ Zhi-Wei Zhou, ${ }^{1}$ Shu-Qiang Yuan, ${ }^{1}$ Yun Wang ${ }^{1},{ }^{4}$ and Yuan-Fang $L i{ }^{1}{ }^{1}$ \\ ${ }^{1}$ Department of Gastric Surgery, Sun Yat-sen University Cancer Center; State Key Laboratory of Oncology in South China; \\ Collaborative Innovation Center for Cancer Medicine, Guangzhou, China \\ ${ }^{2}$ Department of Psychiatry, The Third Affiliated Hospital, Sun Yat-sen University, No. 600 Tianhe Road, Guangzhou, China \\ ${ }^{3}$ Department of Radiation Oncology, Sun Yat-sen University Cancer Center; State Key Laboratory of Oncology in South China; \\ Collaborative Innovation Center for Cancer Medicine; Guangdong Key Laboratory of Nasopharyngeal Carcinoma Diagnosis \\ and Therapy, Guangzhou, China \\ ${ }^{4}$ Department of Hematologic Oncology, Sun Yat-sen University Cancer Center; State Key Laboratory of Oncology in South China; \\ Collaborative Innovation Center for Cancer Medicine, Guangzhou, China
}

Correspondence should be addressed to Yun Wang; 309850291@qq.com and Yuan-Fang Li; liyuanf@sysucc.org.cn

Received 5 October 2019; Revised 19 February 2020; Accepted 25 February 2020; Published 6 May 2020

Academic Editor: Brad Upham

Copyright ( 2020 Run-Cong Nie et al. This is an open access article distributed under the Creative Commons Attribution License, which permits unrestricted use, distribution, and reproduction in any medium, provided the original work is properly cited.

\begin{abstract}
Objectives. To evaluate the efficacy of immuno-oncology combinational therapy (IOCT) versus monotherapy with programmed cell death 1 (PD-1) or PD-ligand 1 (PD-L1) inhibitors or conventional therapies, i.e., non-IOCT, in patients with advanced solid tumors. Methods. We systematically searched the PubMed, Embase, and Cochrane Library databases from January 2015 to October 2018 for eligible studies. We included randomized trials of IOCT with available hazard ratios (HR) for death. The random effects model was used to calculate pooled HR for death; heterogeneity was assessed using $I^{2}$ statistics. The main outcome measure was overall survival (OS). Results. After screening 483 relevant articles, we identified twelve trials comprising 5388 patients for quantitative analysis. IOCT-treated patients had significantly higher tumor response rate (relative risk (RR): 2.51, 95\% confidence interval (CI): 1.82-3.47), prolonged progression-free survival (HR 0.62, 95\% CI: 0.53-0.74), and OS (HR 0.69, 95\% CI: 0.61-0.78), compared with non-IOCT-treated patients. Sensitivity analyses also demonstrated the OS advantage of IOCT across different combination modalities, intervention agents, malignancy types, and PD-L1 expression (all $P<0.05$ ). Notably, there were higher odds of high-grade (grade $\geq 3$ ) adverse events with IOCT (RR: 1.81, 95\% CI: 1.13-2.90), but the risk of treatment-related death (RR: 1.16,95\% CI: 0.84-1.60) was not increased compared with non-IOCT. Conclusions. IOCT is a preferable treatment option over PD-1/PD-L1 inhibitor monotherapy and conventional therapy for patients with advanced solid tumors. However, we should note the increased incidence rate of high-grade AEs in IOCT.
\end{abstract}

\section{Introduction}

Immune checkpoints are a series of coinhibitory and costimulatory receptors and ligands that control the process of immune suppression and evasion of malignant cancer cells, which are known as one of the hallmarks of cancer [1]. The programmed cell death 1 (PD-1) and programmed cell death ligand 1 (PD-L1) axis is one of the most important immune checkpoints as well as a valuable therapeutic target because it not only plays a key role in physiological immune homoeostasis, but also appears to be a means through which cancer cells evade the immune system [2]. The development and application of antibodies targeting PD-1 (nivolumab and pembrolizumab) and PD-L1 (atezolizumab, avelumab, and durvalumab) have advanced the treatment of melanoma [3], nonsmall cell lung cancer (NSCLC) [4], renal cell 
cancer [5], colorectal cancer [6], and head and neck cancer [7]. Currently, PD-1 or PD-L1 inhibitors are being investigated in more than 1000 clinical trials and are licensed to treat a variety of cancers by the U.S. Food and Drug Administration (FDA).

Nonetheless, although immuno-oncology therapy (IOT) is greatly advantageous in that it covers a wide range of tumor types, many shortcomings remain. Principally, the majority of patients could not achieve satisfactory treatment effects from immuno-oncology (IO) monotherapy due to the low overall response rate, varying from $20 \%$ to $40 \%$ [2, 8-13]. Using NSCLC as an example, IO monotherapy only improves the overall survival of a minority of patients that with PD-L1 expression $\geq 50 \%[11,14]$. Additionally, PD1/PD-L1 inhibitors rely heavily on the tumor microenvironment to work; theoretically, only a fraction of patients with inflamed tumor could benefit from immunotherapy, and other immune types such as the immune-desert phenotype and immune-excluded tumors have poor response partly due to the absence of immune effector cells in the tumor microenvironment or obstruction between the immune effector cells and tumor cells [15]. Furthermore, IOT is associated with several immune-related adverse events [16] and requires an extremely high cost, as estimated as more than $£ 234000$ ( $€ 258000 ; \$ 300000)$ per quality adjusted life year [17]. Hence, much remains to be done before IOT can be extensively used in cancer treatment, and an immediate priority is improving the therapeutic efficacy of immunotherapy. To address these issues, substantial clinical trials are underway to explore whether combination with other therapies could improve the treatment effect of IOT.

To date, more than 1100 trials on several combinational modalities, such as IOT plus IOT (namely ipilimumab), chemotherapy, and targeted therapy, are underway for numerous cancer types [18]; initial inspiring results have been achieved with the combinations of IOT plus IOT [19] and IOT plus chemotherapy [20]. Nonetheless, as IOT clinical trials usually require long follow-up duration and large sample sizes to achieve statistical differences and have inconsistent results (both survival outcomes and adverse events [AEs]) among different trials [19-30], it is therefore essential to conduct a meta-analysis to pool the results of the available trials to explore the therapeutic efficacy and safety of IO combination treatment (IOCT) across different tumor types and between IOCT vs. PD-1/PD-L1 inhibitor monotherapy or conventional therapies (non-IOCT) to provide critical and useful information for the clinical utility of IOCT.

\section{Methods}

This study was conducted in compliance with Cochrane Handbook for Systematic Reviews of Interventions recommendations and was reported based on Preferred Reporting Items for Systematic Reviews and Meta-Analyses (PRISMA) statement guidelines [31].

2.1. Search Strategy and Selection Criteria. This is a trial-level meta-analysis. RCN and CBZ conducted a comprehensive systematic search of the Medline (PubMed), Embase, and
Cochrane Library databases from January 2015 to October 2018 with no language restrictions to identify randomized controlled trials (RCT) of IOCT for advanced solid tumors. The main keywords were nivolumab, pembrolizumab, avelumab, atezolizumab, durvalumab, PD-1, PD-L1, checkpoint inhibitors, phase 2 trial, phase 3 trial, and randomized trial (see Supplementary Material (available here)). To be eligible, the RCT had to meet several prespecified criteria: population: enrolled patients with advanced solid tumors irrespective of site; experimental intervention: treated with PD-1 or PD-L1 inhibitors in combination with other treatment modalities irrespective of dosage and duration; control group: treated with anti-PD-1/PD-L1 single agent, ipilimumab, chemotherapy, or targeted therapy; and main outcome: reported outcome of overall survival measured as hazard ratios (HR). We excluded phase 1, nonrandomized phase 2 studies, retrospective, prospective observational cohort, reviews, basic science studies, quality of life studies, case reports, costeffectiveness analyses, commentaries, conference abstracts without published full text original articles, and editorials. Furthermore, we examined the reference lists of all RCT fulfilling the eligibility criteria for any eligible studies missed by the initial search. Discrepancies in the literature search and inclusion were resolved by discussion and consensus.

2.2. Data Extraction and Risk of Bias Assessment. RCN, CBZ, and YSL extracted the reported HR for overall survival and the following clinicopathological characteristics of each eligible trial: article title, accrual period, phase of study, underlying malignancy, line of therapy, treatment regimen, patient number, PD-L1 expression, and median follow-up time. We used the Cochrane Risk of Bias Tool [32] to evaluate the risk of bias of every trial and scored it as high, low, or unclear risk of bias based on the following criteria: random sequence generation, allocation concealment, blinding, incomplete outcome data, and selective reporting.

2.3. Statistical Analysis. The primary endpoint of the present study was overall survival, defined as the time from randomization to death from any cause. The secondary endpoints were progression-free survival (the time from randomization to first RECIST 1.1- (response evaluation criteria in solid tumors-) defined progression or death), objective response rate (the percentage of patients with a confirmed best response of complete response or partial response according to RECIST 1.1), and treatment safety. AEs were graded according to the National Cancer Institute Common Terminology Criteria for Adverse Events, with grade 3, 4, or 5 considered severe. We calculated the treatment effects (HR or relative risk (RR)) of IOCT vs. non-IOCT, with 95\% confidence interval (CI). Statistical heterogeneity between different trials and subgroups were assessed by the Cochrane Q statistic, and the extent of inconsistency contributing to the heterogeneity across different studies was assessed by $I^{2}$ [33]. We considered $I^{2}>50 \%$ to indicate substantial heterogeneity. In the present study, the pooled HRs for death were calculated using the random effects model. Subgroup analyses were conducted based on combination modality, intervention agent, intervention agent target, type of control 


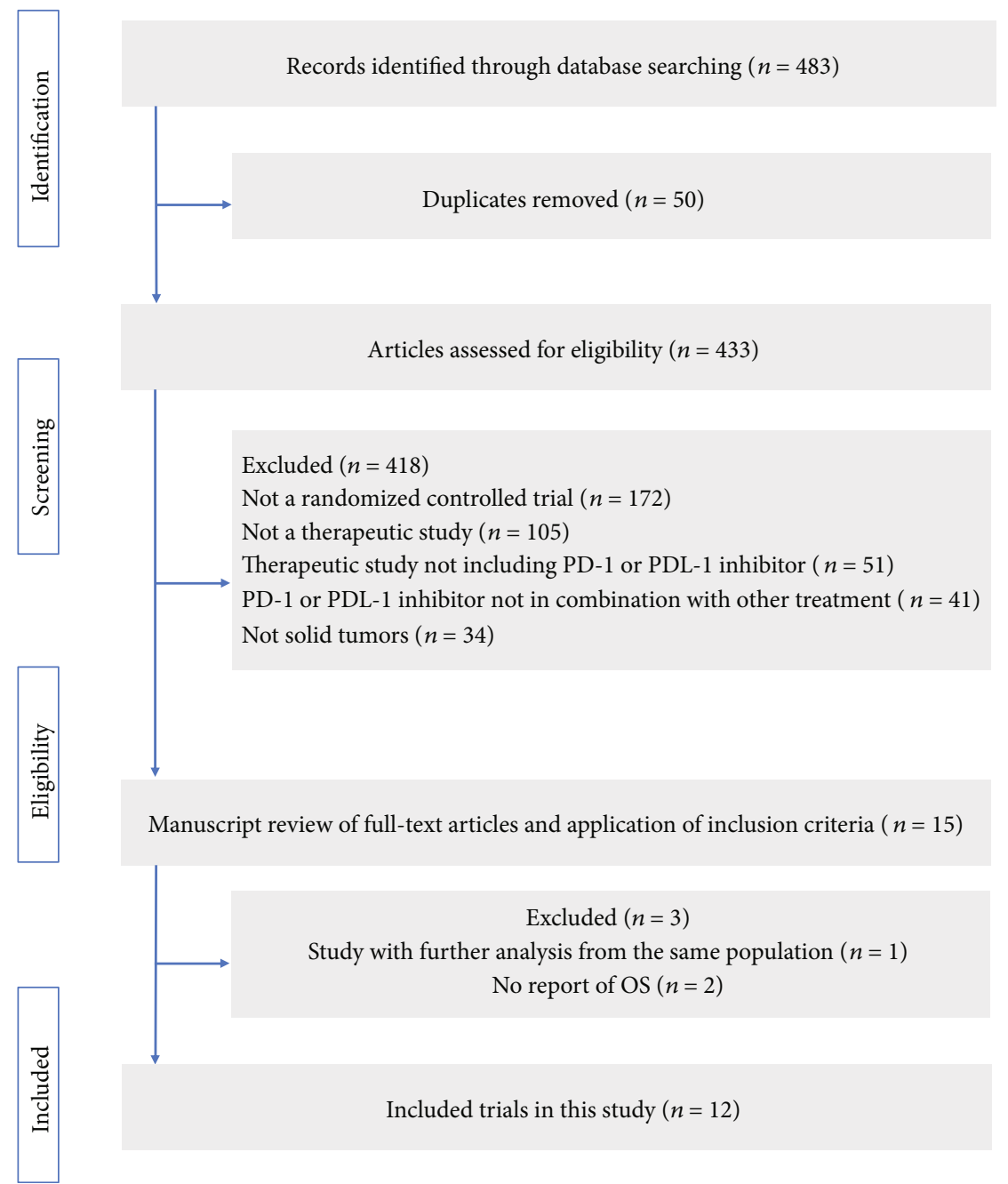

FIGURE 1: Flowchart diagram of study inclusion and exclusion. PD-1: programmed cell death 1; PD-L1: programmed cell death ligand 1; OS: overall survival.

group, malignancy type, and PD-L1 expression. Sensitivity analyses were performed with restriction to large trials (>400 patients) and trials with mature follow-up time (median follow - up $\geq 24$ months).

Potential publication bias was assessed via visual inspection of a funnel plot and evaluated using Begg's regression asymmetry tests [34]. All analyses were performed using Stata version 12.0 (StataCorp, College Station, TX, USA). All tests were two sided; $P<0.05$ was considered statistically significant.

\section{Results}

3.1. Literature Search. After initial systematic literature review, we identified a total of 483 articles on the topic; 468 articles were excluded because they did not meet the inclusion criteria after eligibility screen of the titles and abstracts. We carefully reviewed the full texts of the remaining 15 potentially eligible papers and identified one duplicate report of the same data and two that did not report overall survival. The CheckMate 032 [21] was an open-label, phase 1/2 trial, and we included the two arms of nivolumab $3 \mathrm{mg} / \mathrm{kg}$ plus ipi- limumab $1 \mathrm{mg} / \mathrm{kg}$ (54 patients) and nivolumab $3 \mathrm{mg} / \mathrm{kg}$ (98 patients). Hence, 12 RCT met the inclusion criteria for final analysis [19-30]. Figure 1 details the study selection process.

3.2. Study Characteristics. The 12 eligible RCT included in the present study involved 5388 patients: 2758 (51.2\%) were treated with IOCT, and $2630(48.8 \%)$ were treated with non-IOCT. Table 1 details the characteristics of the RCT. The included studies were all international, multicenter RCT ( 5 phase 2 and 7 phase 3 ) funded by the pharmaceutical industry and published between 2016 and 2018 and provided fundamental evidence for the FDA to license these drugs. Most of the treatment line was first line therapy. The combinational modalities included IOT plus IOT (ipilimumab) (six trials), IOT plus chemotherapy (five trials), and IOT plus chemoradiotherapy (one trial). The CheckMate 067 trial [30] had one IOCT cohort (nivolumab plus ipilimumab group) and two non-IOCT cohorts (nivolumab group and ipilimumab group), leading to 13 comparisons in our analysis. The median follow-up varies from 7.8 months to 38 months. The method quality of the included trials was generally moderate to good; the main issue affecting quality was 


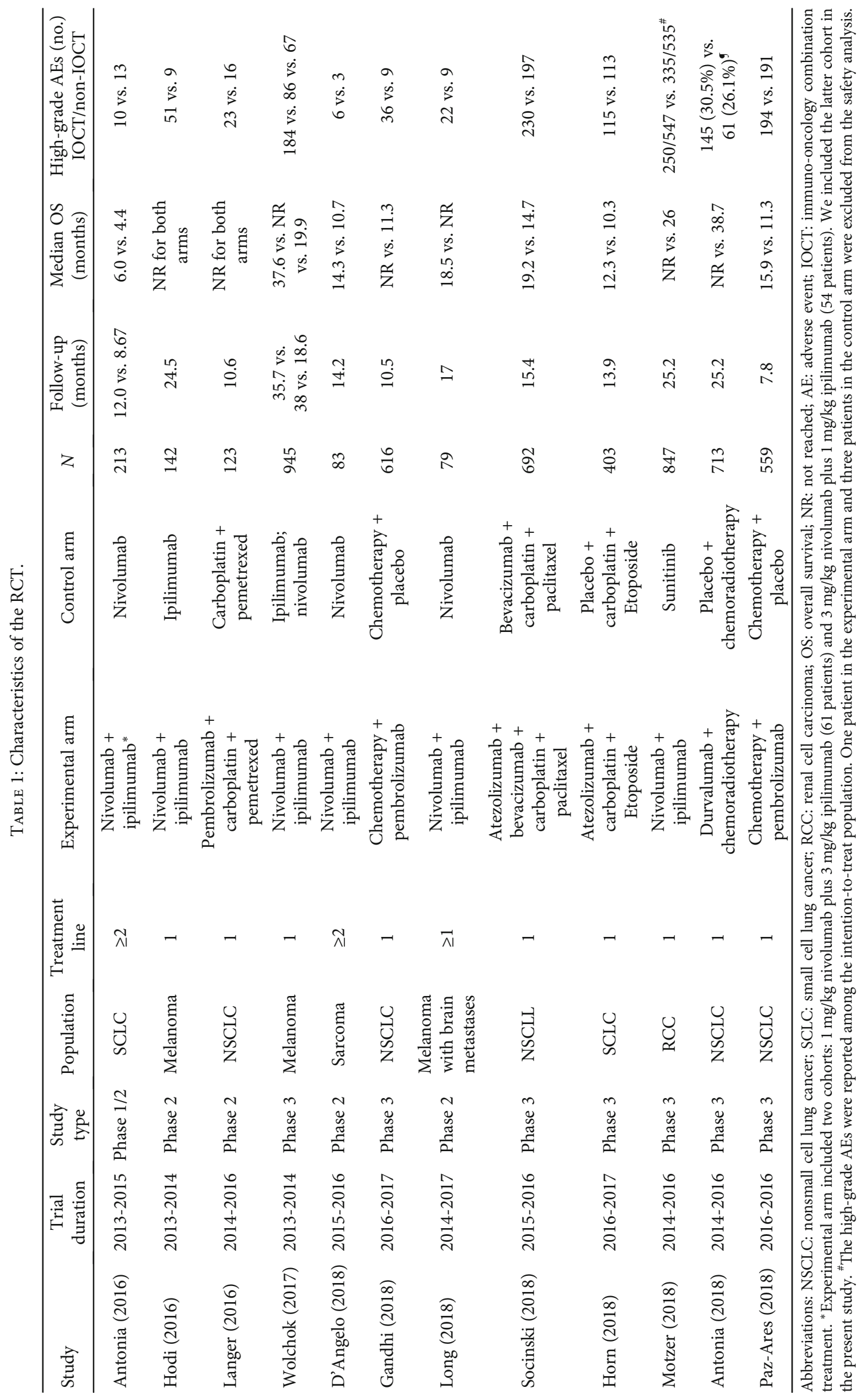




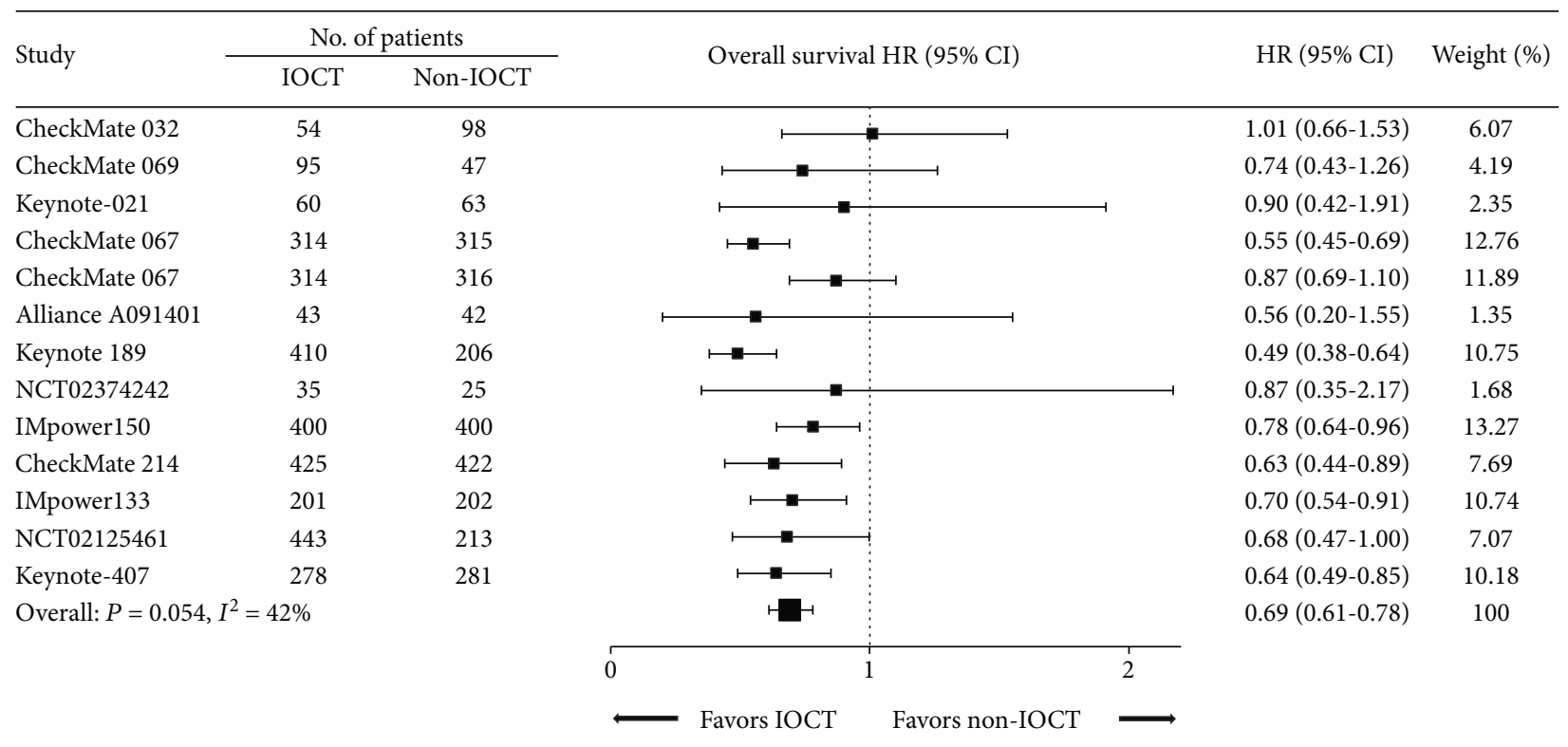

FIGURE 2: Forest plot of HRs comparing overall survival in patients who received IOCT vs. non-IOCT. Studies are listed on the left with respective number of patients of each treatment, $\mathrm{HR}$ with $95 \% \mathrm{CI}$, and weight are on the right. HR: hazard ratio; IOCT: immunooncology combination treatment; CI: confidence interval.

lack of blinding, where six trials were open-label (Supplementary Table 1).

3.3. Therapeutic Efficacy of IOCT for Overall Survival. First, we calculated the treatment effect of IOCT vs. non-IOCT: IOCT-treated patients had significantly reduced risk of death in seven comparisons (HR: $0.49-0.78$, all $P<0.05$; Figure 2) of large sample sizes ( $>400$ participants). Nonetheless, survival benefit was not reached in the other six comparisons (HR: 0.56-1.01, all $P>0.05$; Figure 2), among which five had small sample sizes. Notably, moderate heterogeneity was observed in the overall treatment effect across the 13 comparisons $\left(P=0.054, I^{2}=42.0 \%\right)$; therefore, the random effects model was preferred for the pooled analysis. Overall, IOCT was associated with significantly higher overall response rate (pooled RR: 2.51, 95\% CI: 1.82-3.47, $P<0.001$, Supplementary Figure S1A), prolonged progression-free survival (pooled HR: 0.62, 95\% CI: 0.53-0.74, $P<0.001$; Supplementary Figure S1B), and overall survival (pooled HR: $0.69,95 \%$ CI: $0.61-0.78, P<0.001$; Figure 2 ), compared with non-IOCT.

3.4. Subgroup and Sensitivity Analyses. To further evaluate the therapeutic efficacy of IOCT, we performed subgroup analyses according to the treatment, patient, and trial factors. Mainly, both the combinational modalities of IOT plus IOT (pooled HR: 0.72, 95\% CI: 0.59-0.90, $P=0.003$; Figure 3) and IOT plus chemotherapy (pooled HR: $0.66,95 \%$ CI: $0.57-0.78, P<0.001$; Figure 3 ) showed overall survival benefit for patients. Furthermore, in subgroup analyses stratified by intervention agent, drug target, and control group identified consistent therapeutic efficacy among these subgroups (all pooled $\mathrm{HR}<1.0$; all $P<0.05$ ). Additionally, in subgroups of different cancer types, there was significant survival benefit in patients with different malignancy types (pooled HR for NSCLC, melanoma, and other cancers: $0.66,0.71$, and 0.62 , respectively, all $P<0.030$; Figure 3 ), with the exception of SCLC (pooled HR: 0.81, 95\% CI 0.57-1.15, $P=0.237$, Figure 3), partly due to the limited number of patients involved (555 patients in two comparisons). These findings further indicate that IOCT can reduce risk of death regardless of combinational modality, interventional PD-1/PD-L1 inhibitor, and cancer type.

Next, we explored the IOCT treatment effect in subgroups according to PD-L1 expression. There was overlap among patients with different PD-L1 expression levels due to the multiple thresholds of PD-L1 expression reported in some trials. In summary, IOCT was effective in reducing risk of death in patients with high and low PD-L1 expression (pooled HRs for PD $-\mathrm{L} 1 \geq 50 \%$ (0.51), $\geq 1 \%(0.62),<5 \%$ (0.74), and $<1 \%$ (0.75), respectively, all $P \leq 0.050$; Figure 3), indicating that IOCT was less reliant on pretreatment PD-L1 expression level when combined with other therapies. Interestingly, a tendency for reduction in pooled HR persisted with higher PD-L1 expression.

3.5. Safety of IOCT vs. Non-IOCT. The incidence of AEs of IOCT varies from $74.7 \%$ to $100 \%$, compared to those of IOT of $53.1 \%$ to $100 \%$ (Figure $4(\mathrm{a})$ ). Safety analyses of adverse events showed that IOCT had higher odds of any AEs (pooled RR: $1.84,95 \%$ CI: 1.10-3.07, $P=0.020$, Figure 4(a)). Table 1 presents the incidences of high-grade (grade $\geq 3$ ) AEs of each trial. IOCT had a tendency towards high-grade AEs in the majority of trials compared with non-IOCT; this was more obvious in trials of IOT plus IOT. Overall, IOCT-treated patients had higher odds of high-grade AEs (pooled RR: $1.81,95 \%$ CI: $1.13-2.90, P=0.012$, Figure $4(\mathrm{~b})$ ) compared with non-IOCT-treated patients. Nonetheless, IOCT did not 


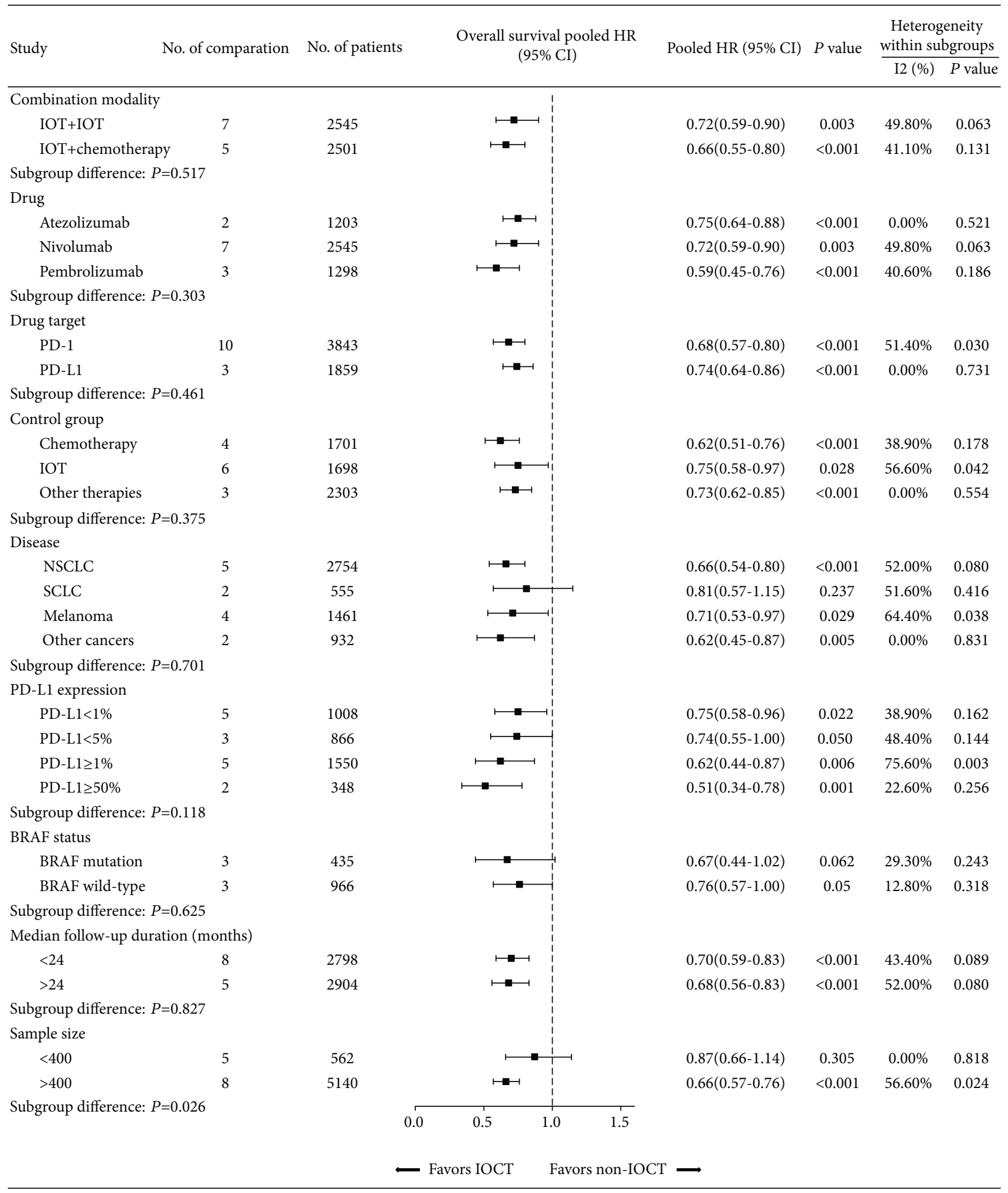

FIGURE 3: Subgroup and sensitivity analyses stratified by treatment, disease, and trial characteristics. HR: hazard ratio; IOCT: immunooncology combination treatment, CI: confidence interval.

increase the risk of treatment-related death compared with non-IOCT (mortality rate, IOCT vs. non-IOCT: $0-8.2 \%$ vs. 0-6.6\%; pooled RR: $1.16,95 \% \mathrm{CI}: 0.84-1.60, P=0.375)$.
3.6. Publication Bias. We did not identify substantial asymmetry in visual inspection of the Begg's funnel plot (Supplementary Figure S2), which the Begg's rank 


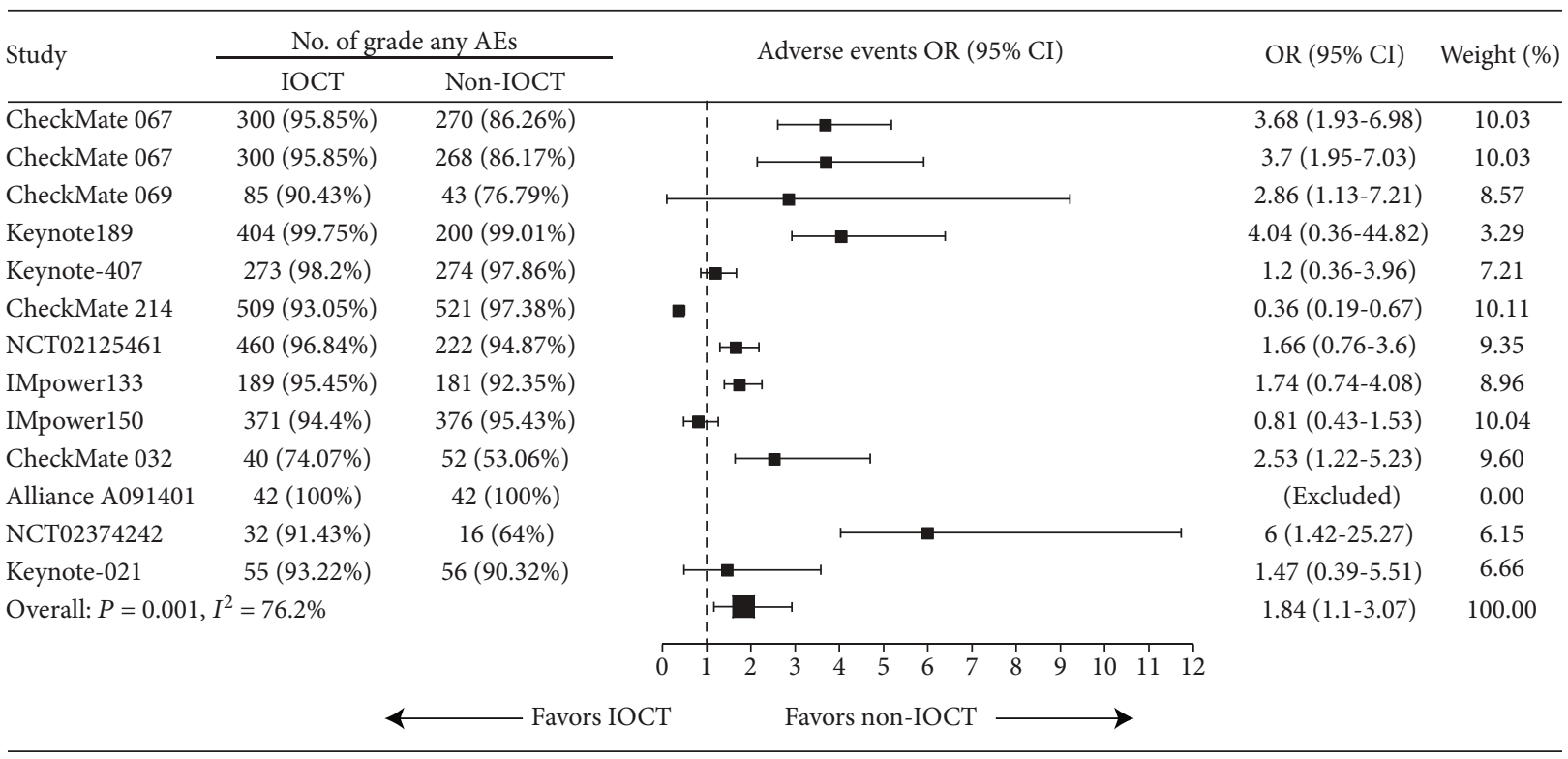

(a)

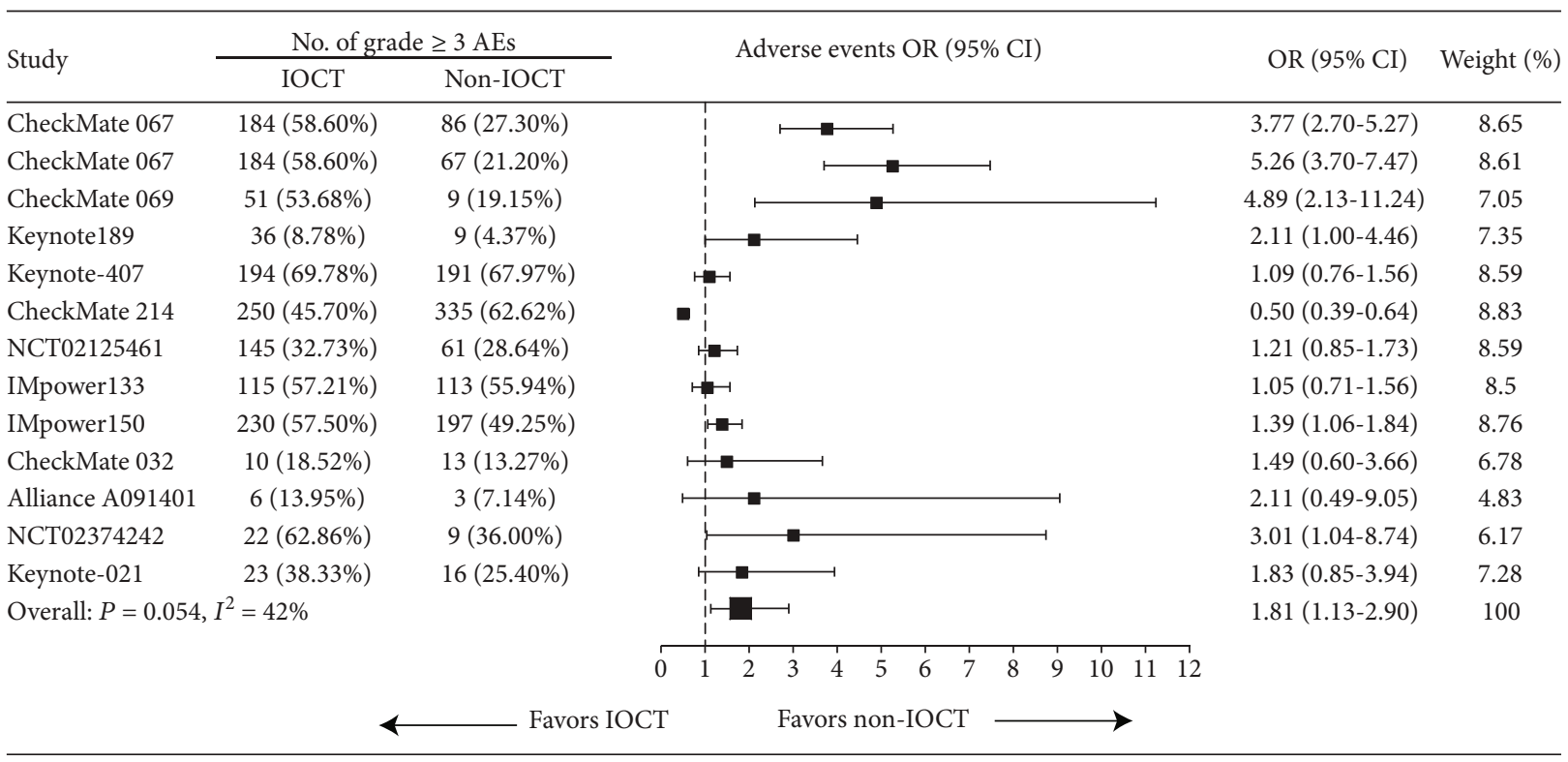

(b)

FIGURE 4: Forest plot of ORs comparing high-grade treatment-related AEs in patients who received IOCT vs. non-IOCT. Studies are listed on the left with respective number of patients of each treatment, OR with 95\% CI and weight are on the right. (a) AEs regardless of the grade; (b) grade $\geq 3$ AEs. OR: odds risk; IOCT: immuno-oncology combination treatment, CI: confidence interval; AE: adverse event.

correlation and Egger linear regression tests confirmed, indicating no evidence of publication bias $(P=0.542)$.

\section{Discussion}

IOCT represents a promising treatment modality for malignancies. Recently, several RCT have reported inspiring survival outcomes in patients who received IOT in combination with other therapies, such as ipilimumab [30], vascular endothelial growth factor (VEGF) inhibitor (bevacizumab) [25], and chemotherapy [28]. To the best of our knowledge, the present study is the first pooled analysis to summarize the therapeutic efficacy and safety of IOCT of these studies. Using the published data from 12 high-quality RCT that comprised 5388 patients with five different types of advanced solid tumors, our pooled analysis reveals that, compared with non-IOCT, IOCT was significantly associated with $38 \%$ reduction in the risk of progression and $31 \%$ reduction in the risk of death. The overall survival advantage of IOCT was identified across different combination modalities, intervention agents, and malignancy types. Interestingly, when combined with other therapies, IOT showed survival benefit for patients with high and low pretreatment PD-L1 expression (pooled HR: 0.51-0.75, all $P \leq 0.050$ ), indicating that IOCT has antitumor activity without the restriction of pretreatment PD-L1 expression. 
Contrary to conventional therapies, such as chemotherapy and targeted therapy, IOT relies heavily on the tumor microenvironment and antitumor immunity, both of which are dynamically altered during tumor microenvironmentimmunity interaction [35-37]. Combining other therapies with IOT may potentially modify the tumor immune microenvironment (such as the abscopal effect of radiotherapy) [38] and promote the antitumor immunity to augment the therapeutic efficacy of IOT [39]. Currently, the most promising combination modalities for PD-1/PD-L1 inhibitors include other immune checkpoint inhibitors (namely CTLA-4 inhibitors), chemotherapy, and targeted therapies [40]. The rationales behind these combinations are multifactorial and include activating $\mathrm{T}$ cells by regulating different signaling pathways (the CTLA-4 checkpoint is critical for $\mathrm{T}$ cell priming and activation, whereas PD-1 blocks effector $\mathrm{T}$ cell responses in tissues), enhancing tumor antigen expression, exposing more new antigen mutations and higher mutation burdens, inducing PD-L1 expression, augmenting $\mathrm{T}$ cell infiltration around the metastatic sites, and producing a more favorable tumor microenvironment [1, 41]. Currently, a substantial number of trials have reported excellent outcomes of these combination modalities in several cancer types [21-30], which facilitated FDA approval of the nivolumab plus ipilimumab combination as firstline treatment for BRAF V600 wild-type unresectable or metastatic melanoma [42] and pembrolizumab plus pemetrexed and carboplatin as first-line treatment for metastatic NSCLC, irrespective of PD-L1 expression [28]. In the present study, pooled analysis of the 12 RCT also showed consistent benefit on tumor response, progression-free survival, and overall survival in IOCT-treated patients. The overall survival benefit of IOCT was identified across different combination modalities. These findings provide critical evidence for the clinical utility of IOCT in treating cancer, and we propose that trials with more combinational modalities in extensive tumor types be conducted.

Our study provides important evidence for broadening the scope of IOT application in even patients with low PDL1 expression. This is of great clinical significance, as PD-1 and PD-L1 inhibitor first-line monotherapy is currently limited to patients with high PD-L1 expression, while most patients, such as those with metastatic NSCLC, have tumors with low or negative PD-L1 expression [43]. Numerous studies have examined the role of PD-L1 expression as a predictive biomarker of tumor response; nonetheless, its predictive value remains unclear due to the different cut-off values (e.g., $1 \%, 5 \%, 10 \%$, and $50 \%$ ) reported for the definition of PDL1 positivity or negativity, which is further compounded by the possibility of interlaboratory variation [23-25, 43]. IOCT may help to overcome these issues through the immunomodulatory effect; inducing PD-L1 expression in tumor cells and exposing tumor antigens to immunocytes $[1,39,41]$. To validate the synergistic effect of immunotherapy and combinational therapies, we relied on a large dataset of 5388 cases, for all of whom the outcomes of a well-defined endpoint (overall survival) was reported. Through pooled analyses, we observed that although patients with high PD-L1 expression did better, those with low PD-L1 expression also benefit from the IOCT. We believe that the favorable overall survival achieved in the patients with low PD-L1 expression is mainly due to the immunomodulatory effect of the combinational therapies, which still rely on the biological function of the PD-1 or PD-L1 pathway and the complicated interaction between cancer cells and the immune system.

The AEs related to the PD-1/PD-L1 checkpoint inhibitors are generally considered well-tolerated and manageable, particularly when compared to the toxicity profile of other immunotherapy drugs such as CTLA-4 inhibitors and chemotherapy [44]. In IOCT, although the profile of AEs is similar to that observed in monotherapy and was generally reversible, the overall incidence and the incidence of high-grade AEs increased significantly. Therefore, we should be aware of the incidence and appropriate management of severe AEs caused by IOCT, especially the immune-related AEs such as immunerelated myocarditis [45] and meningitis [46], when conducting a clinical trial or in conventional clinical use.

Our study has some notable limitations. First, there is moderate heterogeneity among the eligible studies $(P=0.054$ , $\left.I^{2}=42.0 \%\right)$. However, the actual heterogeneity could be higher than the statistical heterogeneity. We believed that the heterogeneity is derived from the multiple intervention modalities (combinational therapies and PD-1/PD-L1 inhibitors) and various tumor types (NSCLC, melanoma, sarcoma, and others); therefore, although we performed subgroup analyses to mitigate that limitation, high heterogeneity should be noted when interpreting our results. Then, some of the eligible trials had a small number of participants and short follow-up duration, which may have resulted in fairly wide CIs of HRs for the treatment effects, thereby confounding our pooled HR results. Nonetheless, in sensitivity analyses restricted to large-scale trials (>400 participants) and trials with a long follow-up duration ( $>24$ months), consistent survival advantages (large-scale trials: pooled HR: $0.66, P<0.001$; long follow-up duration trials: pooled HR: $0.68, P<0.001$; Figure 3) persisted. Next, this is a meta-analysis at trial level rather than individual level, the detailed information on patients that may impact the efficacy of immunotherapy, such as patient demographic (age and sex), was not available. Lastly, some trials had a crossover design, which could have weakened the treatment effect of IOCT. Nonetheless, we observed favorable results in this meta-analysis.

In conclusion, compared with $\mathrm{PD}-1 / \mathrm{PD}-\mathrm{L} 1$ inhibitor monotherapy and conventional therapies, IOCT significantly prolonged overall survival in patients with advanced solid tumors regardless of cancer type and PD-L1 expression. However, we should note the increased incidence rate of high-grade AEs in IOCT. The present study findings could also aid clinicians in the clinical practice use of IOCT.

\section{Data Availability}

All data used to support the findings of this study are included within the article.

\section{Conflicts of Interest}

The authors declare that they have no conflicts of interest. 


\section{Authors' Contributions}

Run-Cong Nie, Chong-Bang Zhao, Xiao-Wei Xia, and YingShan Luo contributed equally to this work. Yun Wang and Yuan-Fang Li are cosenior authors.

\section{Supplementary Materials}

PubMed search terms. Supplementary Table 1: risk of bias of the included trials. Supplementary Figure S1: forest plot of $\mathrm{RR}$ comparing overall response (A), and HR comparing progression-free survival (B) in patients who received IOCT vs. non-IOCT. RR: relative risk; HR: hazard ratio; IOCT: immuno-oncology combination treatment, CI: confidence interval. Supplementary Figure S2: Begg's funnel plot for publication bias test. Each circle represents a separate study for indicated association. Horizontal line, mean effect size. HR: hazard ratio. (Supplementary Materials)

\section{References}

[1] D. Hanahan and R. A. Weinberg, "Hallmarks of cancer: the next generation," Cell, vol. 144, no. 5, pp. 646-674, 2011.

[2] C. Sun, R. Mezzadra, and T. N. Schumacher, "Regulation and function of the PD-L1 checkpoint," Immunity, vol. 48, no. 3, pp. 434-452, 2018.

[3] N. Margolis, E. Markovits, and G. Markel, "Reprogramming lymphocytes for the treatment of melanoma: from biology to therapy," Advanced Drug Delivery Reviews, vol. 141, pp. 104124, 2019.

[4] L. Corrales, K. Scilla, C. Caglevic, K. Miller, J. Oliveira, and C. Rolfo, "Immunotherapy in lung cancer: a new age in cancer treatment," Advances in Experimental Medicine and Biology, vol. 995, pp. 65-95, 2018.

[5] R. Flippot, B. Escudier, and L. Albiges, "Immune checkpoint inhibitors: toward new paradigms in renal cell carcinoma," Drugs, vol. 78, no. 14, pp. 1443-1457, 2018.

[6] D. Basile, S. K. Garattini, M. Bonotto et al., "Immunotherapy for colorectal cancer: where are we heading?," Expert Opinion on Biological Therapy, vol. 17, no. 6, pp. 709-721, 2017.

[7] S. Wiegand, G. Wichmann, and A. Dietz, "Perspectives of induction with chemo and/or immune check point inhibition in head and neck organ preservation treatment," Frontiers in Oncology, vol. 9, 2019.

[8] R. J. Motzer, B. I. Rini, D. F. McDermott et al., "Nivolumab for metastatic renal cell carcinoma: results of a randomized phase II trial," Journal of Clinical Oncology, vol. 33, no. 13, pp. 14301437, 2015.

[9] G. Giaccone, C. Kim, J. Thompson et al., "Pembrolizumab in patients with thymic carcinoma: a single-arm, single-centre, phase 2 study," The Lancet Oncology, vol. 19, no. 3, pp. 347$355,2018$.

[10] C. Robert, G. V. Long, B. Brady et al., "Nivolumab in previously untreated melanoma without BRAF mutation," The New England Journal of Medicine, vol. 372, no. 4, pp. 320330, 2015.

[11] H. Borghaei, L. Paz-Ares, L. Horn et al., "Nivolumab versus docetaxel in advanced nonsquamous non-small-cell lung cancer," The New England Journal of Medicine, vol. 373, no. 17, pp. 1627-1639, 2015.
[12] M. J. Overman, R. McDermott, J. L. Leach et al., "Nivolumab in patients with metastatic DNA mismatch repair-deficient or microsatellite instability-high colorectal cancer (CheckMate 142): an open-label, multicentre, phase 2 study," The Lancet Oncology, vol. 18, no. 9, pp. 1182-1191, 2017.

[13] R. L. Ferris, G. Blumenschein Jr., J. Fayette et al., "Nivolumab for recurrent squamous-cell carcinoma of the head and neck," The New England Journal of Medicine, vol. 375, no. 19, pp. 1856-1867, 2016.

[14] M. Reck, D. Rodríguez-Abreu, A. G. Robinson et al., "Pembrolizumab versus chemotherapy for PD-L1-positive non-smallcell lung cancer," The New England Journal of Medicine, vol. 375, no. 19, pp. 1823-1833, 2016.

[15] D. S. Chen and I. Mellman, "Elements of cancer immunity and the cancer-immune set point," Nature, vol. 541, no. 7637, pp. 321-330, 2017.

[16] S. Baxi, A. Yang, R. L. Gennarelli et al., "Immune-related adverse events for anti-PD-1 and anti-PD-L1 drugs: systematic review and meta-analysis," BMJ, vol. 360, 2018.

[17] J. Wang, B. Chmielowski, J. Pellissier, R. Xu, K. Stevinson, and F. X. Liu, "Cost-effectiveness of pembrolizumab versus ipilimumab in ipilimumab-naïve patients with advanced melanoma in the United States," Journal of Managed Care of Specialty Pharmacy, vol. 23, no. 2, pp. 184-194, 2017.

[18] J. Tang, A. Shalabi, and V. M. Hubbard-Lucey, "Comprehensive analysis of the clinical immuno-oncology landscape," Annals of Oncology, vol. 29, no. 1, pp. 84-91, 2018.

[19] R. J. Motzer, N. M. Tannir, D. F. McDermott et al., "Nivolumab plus ipilimumab versus sunitinib in advanced renal-cell carcinoma," The New England Journal of Medicine, vol. 378, no. 14, pp. 1277-1290, 2018.

[20] L. Horn, A. S. Mansfield, A. Szczęsna et al., "First-line atezolizumab plus chemotherapy in extensive-stage small-cell lung cancer," The New England Journal of Medicine, vol. 379, no. 23, pp. 2220-2229, 2018.

[21] S. J. Antonia, J. A. López-Martin, J. Bendell et al., "Nivolumab alone and nivolumab plus ipilimumab in recurrent small-cell lung cancer (CheckMate 032): a multicentre, open-label, phase 1/2 trial," The Lancet Oncology, vol. 17, no. 7, pp. 883-895, 2016.

[22] F. S. Hodi, J. Chesney, A. C. Pavlick et al., "Combined nivolumab and ipilimumab versus ipilimumab alone in patients with advanced melanoma: 2-year overall survival outcomes in a multicentre, randomised, controlled, phase 2 trial," The Lancet Oncology, vol. 17, no. 11, pp. 1558-1568, 2016.

[23] C. J. Langer, S. M. Gadgeel, H. Borghaei et al., "Carboplatin and pemetrexed with or without pembrolizumab for advanced, non-squamous non-small-cell lung cancer: a randomised, phase 2 cohort of the open-label KEYNOTE-021 study," The Lancet Oncology, vol. 17, no. 11, pp. 14971508, 2016.

[24] G. V. Long, V. Atkinson, S. Lo et al., "Combination nivolumab and ipilimumab or nivolumab alone in melanoma brain metastases: a multicentre randomised phase 2 study," The Lancet Oncology, vol. 19, no. 5, pp. 672-681, 2018.

[25] M. A. Socinski, R. M. Jotte, F. Cappuzzo et al., "Atezolizumab for first-line treatment of metastatic nonsquamous NSCLC," The New England Journal of Medicine, vol. 378, no. 24, pp. 2288-2301, 2018.

[26] S. J. Antonia, A. Villegas, D. Daniel et al., "Overall survival with durvalumab after chemoradiotherapy in stage III 
NSCLC," New England Journal of Medicine, vol. 379, no. 24, pp. 2342-2350, 2018.

[27] S. P. D'Angelo, M. R. Mahoney, B. A. van Tine et al., "Nivolumab with or without ipilimumab treatment for metastatic sarcoma (Alliance A091401): two open-label, non-comparative, randomised, phase 2 trials," The Lancet Oncology, vol. 19, no. 3, pp. 416-426, 2018.

[28] L. Gandhi, D. Rodríguez-Abreu, S. Gadgeel et al., "Pembrolizumab plus chemotherapy in metastatic non-small-cell lung cancer," The New England Journal of Medicine, vol. 378, no. 22, pp. 2078-2092, 2018.

[29] L. Paz-Ares, A. Luft, D. Vicente et al., "Pembrolizumab plus chemotherapy for squamous non-small-cell lung cancer," The New England Journal of Medicine, vol. 379, no. 21, pp. 2040-2051, 2018.

[30] J. D. Wolchok, V. Chiarion-Sileni, R. Gonzalez et al., "Overall survival with combined nivolumab and ipilimumab in advanced melanoma," New England Journal of Medicine, vol. 377, no. 14, pp. 1345-1356, 2017.

[31] A. Liberati, D. G. Altman, J. Tetzlaff et al., "The PRISMA statement for reporting systematic reviews and meta-analyses of studies that evaluate healthcare interventions: explanation and elaboration," BMJ, vol. 339, p. b2700, 2009.

[32] J. P. T. Higgins, D. G. Altman, P. C. Gotzsche et al., "The Cochrane Collaboration's tool for assessing risk of bias in randomised trials," BMJ, vol. 343, p. d5928, 2011.

[33] J. P. Higgins, S. G. Thompson, J. J. Deeks, and D. G. Altman, "Measuring inconsistency in meta-analyses," BMJ, vol. 327, no. 7414, pp. 557-560, 2003.

[34] C. B. Begg and M. Mazumdar, "Operating characteristics of a rank correlation test for publication bias," Biometrics, vol. 50, no. 4, pp. 1088-1101, 1994.

[35] A. Ribas and J. D. Wolchok, "Cancer immunotherapy using checkpoint blockade,” Science, vol. 359, no. 6382, pp. 13501355, 2018.

[36] T. F. Gajewski, H. Schreiber, and Y. X. Fu, "Innate and adaptive immune cells in the tumor microenvironment," Nature Immunology, vol. 14, no. 10, pp. 1014-1022, 2013.

[37] M. S. Rooney, S. A. Shukla, C. J. Wu, G. Getz, and N. Hacohen, "Molecular and genetic properties of tumors associated with local immune cytolytic activity," Cell, vol. 160, no. 1-2, pp. 48-61, 2015.

[38] V. Longo, O. Brunetti, A. Azzariti et al., "Strategies to improve cancer immune checkpoint inhibitors efficacy, other than abscopal effect: a systematic review," Cancers, vol. 11, no. 4, p. 539, 2019.

[39] B. Melosky, Q. Chu, R. A. Juergens et al., "Breaking the biomarker code: PD-L1 expression and checkpoint inhibition in advanced NSCLC," Cancer Treatment Reviews, vol. 65, pp. 65-77, 2018.

[40] M. F. Sanmamed and L. Chen, "A paradigm shift in cancer immunotherapy: from enhancement to normalization," Cell, vol. 175, no. 2, pp. 313-326, 2018.

[41] J. Peng, J. Hamanishi, N. Matsumura et al., "Chemotherapy induces programmed cell death-ligand 1 overexpression via the nuclear factor- $\kappa \mathrm{B}$ to foster an immunosuppressive tumor microenvironment in ovarian cancer," Cancer Research, vol. 75, no. 23, pp. 5034-5045, 2015.

[42] M. A. Postow, J. Chesney, A. C. Pavlick et al., "Nivolumab and ipilimumab versus ipilimumab in untreated melanoma," The
New England Journal of Medicine, vol. 372, no. 21, pp. 2006 2017, 2015.

[43] J. Brahmer, K. L. Reckamp, P. Baas et al., "Nivolumab versus docetaxel in advanced squamous-cell non-small-cell lung cancer," The New England Journal of Medicine, vol. 373, no. 2, pp. 123-135, 2015.

[44] J. R. Brahmer, S. S. Tykodi, L. Q. M. Chow et al., "Safety and activity of anti-PD-L1 antibody in patients with advanced cancer," New England Journal of Medicine, vol. 366, no. 26, pp. 2455-2465, 2012.

[45] S. S. Mahmood, M. G. Fradley, J. V. Cohen et al., "Myocarditis in patients treated with immune checkpoint inhibitors," Journal of the American College of Cardiology, vol. 71, no. 16, pp. 1755-1764, 2018.

[46] P. C. Pan and A. Haggiagi, "Neurologic immune-related adverse events associated with immune checkpoint inhibition," Current Oncology Reports, vol. 21, no. 12, 2019. 